\title{
Análisis de un instrumento estandarizado para la evaluación de la comprensión lectora a partir de un modelo psicolingüístico*
}

\author{
Analysis of a Standardized Instrument for Reading Comprehension Assessment from a \\ Psycholinguistic Model
Análise de um instrumento padronizado para a avaliação da compreensão leitora a partir de um modelo psicolinguístico

\section{Angie Neira Martínez, ${ }^{\mathrm{a}}$ Ginette Castro Yáñez ${ }^{\mathrm{b}}$}

\author{
${ }^{a}$ Universidad de Concepción, Facultad de Humanidades, Departamento de Español. Telf.: (41) 2204313. \\ Correo electrónico: angieneira@udec.cl \\ b Universidad Católica de Temuco, Facultad de Artes y Humanidades, Departamento de Lenguas y \\ Traducción. Telf.: (45) 2205301. Correo electrónico: gcastro@uct.cl
}

\begin{abstract}
RESUMEN
La lectura eficaz se ha constituido en uno de los desafíos fundamentales del sistema educacional chileno, luego que diversas mediciones internacionales mostraran escasos logros en la competencia lectora de niños y jóvenes. En este contexto, existen instrumentos de evaluación del nivel lector, uno de ellos es CLP, que es aplicada para el diagnóstico de la habilidad lectora y la guía de consecuentes acciones remediales. El objetivo de este trabajo es analizar los niveles 4 y 8 de CLP desde un modelo psicolingüístico de la evaluación de la comprensión lectora para así determinar los niveles de comprensión que ésta evalúa. Dicho análisis evidencia que esta prueba mide, principalmente, la dimensión textual de la comprensión lectora en sus niveles más bajos, dejando de lado las dimensiones crítica y pragmática. Se hace necesaria la actualización de esta prueba y el diseño de nuevos instrumentos que midan la lectura de manera integral.
\end{abstract}

Palabras clave: comprensión lectora, evaluación de la comprensión, niveles de comprensión.

\begin{abstract}
Effective reading has become one of the fundamental challenges of the Chilean educational system after international assessment results showed children and young people had poor reading competence. Regarding reading assessment, there are instruments such as CLP (Progressive Linguistic Complexity), which diagnoses reading skills problems and provides remedial actions accordingly. The purpose of this study is to analyze, from a psycholinguistic approach to the reading comprehension, levels 4 and 8 of CLP so as to determine the comprehension levels that it assesses. The analysis shows that this test mainly measures the textual dimension of reading comprehension at its lowest levels, ignoring the pragmatic and critical dimensions. Updating this test and designing new instruments for more wholesome reading measurements become necessary.
\end{abstract}

Key words: reading comprehension, comprehension assessment, comprehension levels.

\section{RESUMO}

A leitura eficaz transformou-se em um dos desafios fundamentais do sistema educacional chileno, assim que diversos resultados internacionais de aferição mostraram escassos êxitos na competência de leitura de crianças e jovens. Neste contexto, existem instrumentos de avaliação do nível de leitura, sendo a CLP um deles, cujos resultados visam diagnosticar a habilidade de leitura e orientar consequentes ações com vistas à melhoria do desempenho. O objetivo

* Este trabajo ha sido desarrollado en el marco del proyecto Fondef D08i1179, "Desarrollo de una prueba validada y normada para el diagnóstico de la comprensión lectora en el sistema escolar chileno". 
de este trabalho é analisar os níveis 4 e 8 da CLP a partir de um enfoque psicolinguístico da avaliação da compreensão de leitura para, assim, determinar os níveis de compreensão que este instrumento avalia. Esta análise demonstra que a prova mede, principalmente, a dimensão textual da compreensão de leitura em seus níveis mais baixos, deixando de lado as dimensões crítica e pragmática. Faz-se necessária a atualização de esta prova e a criação de novos instrumentos que meçam a leitura de maneira integral.

Palavras chave: compreensão de leitura, avaliação da compreensão, níveis de compreensão.

\section{INTRODUCCIÓN}

La lectura es una habilidad fundamental en el desarrollo de una persona, no solo a nivel académico, sino que en todas las actividades de la vida diaria. En la niñez, aprender a leer cobra gran relevancia, lo que implica, en un comienzo, decodificar los textos escritos para, más tarde, comprender su contenido. Sin embargo, la comprensión lectora no es siempre lograda del todo (OCDE, 2009, MINEDUC, 2010, 2011), por lo que su medición se vuelve necesaria en las distintas etapas escolares.

En Chile, se ha producido una serie de instrumentos estandarizados para medir el nivel de comprensión lectora en escolares. Uno de ellos es la prueba de comprensión lectora de complejidad lingüística progresiva (CLP), diseñada por Felipe Alliende, Mabel Condemarín y Neva Milicic (2004), la que en su primera versión, en los años 80, incluyó cinco niveles correspondientes a los primeros cinco cursos de educación general básica y en su segunda versión, en los años 90, integró los niveles seis a ocho. Esta prueba es, actualmente, aplicada en diversos colegios y escuelas del país, por lo que se puede afirmar que es un instrumento que ha evaluado el nivel de comprensión lectora de una gran cantidad de niños en las últimas décadas.

El objetivo de este trabajo es determinar los niveles de comprensión lectora que mide CLP y su pertinencia como herramienta de diagnóstico para la enseñanza. Para ello, se analizarán dos niveles de la prueba CLP: el 4, correspondiente a cuarto año básico, y el 8, correspondiente a octavo año básico. Se han seleccionado estos niveles porque son clave en el sistema de enseñanza, ya que cada uno corresponde a la finalización de un ciclo escolar. Para el análisis, se clasificaron las preguntas de las dos formas de cada nivel de acuerdo con el modelo de evaluación de la comprensión lectora de Riffo y Véliz (Riffo y Véliz, 2011; Véliz y Riffo, 1993) clasificación que se contrapuso a lo declarado por los autores de CLP.

\section{MARCO TEÓRICO}

\subsection{MODELOS DE COMPRENSIÓN LECTORA}

\subsubsection{Modelo de construcción e integración de Kintsch}

La teoría de construcción - integración de Walter Kintsch (1998) explica, a través de un modelo mixto, el fenómeno de la comprensión del discurso. Es considerada un modelo mixto dado que propone la conjunción de representaciones conexionistas y simbólicas, unión poco tradicional en el área de la psicolingüística. A lo largo del tiempo, ha sido ampliamente aceptada y valorada como no controversial, puesto que propone 
una descripción completa del procesamiento cognitivo y de las representaciones mentales involucradas en el proceso de la comprensión lectora.

Esta teoría se distingue porque aborda procesos psicológicos: describe las etapas del procesamiento que llevan a un resultado, esto es, las representaciones mentales del texto leído. Otro aspecto importante de esta teoría es que da cuenta de cómo, para llegar a la comprensión, se pone en juego una serie de elementos para llegar a una configuración estable (Kintsch, 1998).

El modelo describe la comprensión en función de dos procesos principales: uno de construcción, que se produce de abajo hacia arriba y de forma rápida, automática y caótica; y otro de integración, en el que se seleccionan los elementos que se relacionan y se desactivan aquéllos que son irrelevantes. En otras palabras, "a context - insensitive construction process is followed by a constraint - satisfaction, or integration, process that yields if all goes well, an orderly mental structure out of initial chaos" (Kintsch, 1998: 5).

Los niveles propuestos por este modelo son:

a. Nivel lingüístico, o de procesamiento de palabras y frases. En este nivel están involucradas las habilidades de decodificación, reconocimiento de palabras y análisis sintáctico (parsing).

b. Nivel semántico. En este nivel se encuentra el análisis de la micro y la macro estructura, las que determinan el significado del texto. La primera está formada por una compleja red de proposiciones interrelacionadas, que psicológicamente se construye de acuerdo a la relación sintáctica entre las palabras en el texto y a la relación de coherencia entre proposiciones. La segunda se forma al reconocer las ideas globales del texto y la interrelación entre ellas. La micro y la macroestructura juntas forman el texto base, que corresponde a lo que explícitamente se expresa en el texto, lo que será suficiente solo para reproducirlo.

c. Modelo de situación. Éste es un modelo mental de la situación descrita en el texto y requiere de la integración entre la información entregada por el texto, los conocimientos previos del lector y sus metas para comprender. Esta representación permite lograr una comprensión profunda del texto. La construcción del modelo de situación no depende sólo del dominio verbal, sino que se extiende a imágenes, emociones, experiencias personales, etc.

Kintsch y Rawson (2005) destacan el papel del texto base y del modelo de situación en la comprensión lectora. En el primero, se forman redes de información que los sujetos deben ordenar para integrarla y darle coherencia a la lectura. El segundo es la clave de la comprensión, ya que si un lector solo comprende a nivel de texto base, su comprensión será muy limitada y superficial. Para comprender a cabalidad un texto, es necesaria la construcción de un modelo de situación, ya que solo de esta manera pueden integrarse conocimientos previos y nuevos.

\subsubsection{Modelo de comprensión lectora de Perfetti}

El modelo planteado por Perfetti (Perfetti, 1999; Perfetti et al., 2007) contempla dos clases mayores de procesamiento: la identificación de palabras y la participación de mecanismos de procesamiento de lenguaje que ensamblan estas palabras en mensajes. Los procesos previamente mencionados 
(...) provide contextually appropriate word meanings, parse words strings into constituents, and provide inferential integration of sentence information into more complete representations of extended text. These representations are not the result of exclusively linguistic processes, but are critically enhanced by other knowledge sources (Perfetti et al., 2007: 229)

Para los autores, la única diferencia entre la comprensión del lenguaje escrito y la del hablado es la palabra impresa.

Este modelo clasifica los factores que inciden en la comprensión lectora en de más alto nivel y de más bajo nivel. Entre los de más alto nivel se destacan tres: sensibilidad a la estructura de la historia, hacer inferencias y monitoreo de la comprensión. Entre los factores de bajo nivel se encuentran los que los autores llaman mecanismos lingüístico - conceptuales de la comprensión, es decir, los procesos que convierten las oraciones en su significado proposicional.

a) Factores de más alto nivel

Al hacer referencia al primer factor de alto nivel, sensibilidad a la estructura de la historia, es necesario mencionar que el género discursivo incide poderosamente en la comprensión de un texto, ya que las características genéricas (como la trama discursiva predominante, los estilos lingüísticos, el nivel de especialización, etc.) pueden presentar diversos problemas al lector. Se habla de la sensibilidad a la estructura de la historia dado que los cuentos (o historias simples) han sido los que más atención han captado entre los investigadores.

El segundo factor, hacer inferencias, es un requisito para una comprensión de lectura profunda. Para Perfetti et al. (2007), las inferencias más necesarias para la comprensión son aquéllas que permiten establecer la coherencia en el texto. El tercer factor, monitoreo de la comprensión, permite al lector verificar su nivel de entendimiento y realizar acciones de reparación cuando no está entendiendo (como releer). Los lectores poco hábiles tienen un bajo desempeño en el monitoreo de su comprensión.

\section{b) Factores de más bajo nivel}

El primer factor es el procesamiento sintáctico. A pesar de que la gramática de la lengua nativa del individuo se adquiere naturalmente, es de esperar que las diferencias en las habilidades lingüísticas desemboquen en diferencias individuales en comprensión. De acuerdo a los diversos estudios (como los de Crain y Shankweiler, 1988; Carpenter et al., 1994; Cit. en Perfetti et al., 2007), los problemas de parsing sintáctico pueden ser causados por limitaciones de procesamiento (en especial, de la memoria operativa) más que por una falta de conocimientos sintácticos.

El segundo factor es la identificación y comprensión de las palabras. La relación causal entre comprensión y vocabulario parece ser recíproca. Esto es, una palabra puede ser inferida desde el contexto, gracias a la habilidad de comprensión; por otra parte, la habilidad de acceder al significado de las palabras de un texto en particular es esencial para la comprensión del mismo. Este factor es crítico, ya que si un lector menos hábil tiene un sistema léxico débil, ello se reflejará en el funcionamiento de su sistema semántico.

En un nivel más bajo, se encuentran la identificación de palabras, la decodificación y la conciencia fonológica. Estos factores no son menos importantes en el proceso de 
comprensión lectora debido a que, como se ha comprobado, la conciencia fonológica predice la comprensión en niños pequeños, independientemente de la memoria operativa (Leather y Henry, 1994, Cit. en Perfetti et al., 2007). Además, se ha establecido que la habilidad de identificación de palabras y la de comprensión se desarrollan en apoyo mutuo (Perfetti, 1985, 1991). Uno de los factores fundamentales para una lectura fluida es el desarrollo de representaciones de palabras de alta calidad, lo que permite que la comprensión del niño esté menos limitada a la identificación de palabras y más influenciada por otros factores. Esto se refleja en las habilidades de lectura en la adultez, ya sea en una limitación persistente de la identificación de palabras en la comprensión o por el fortalecimiento de ambas habilidades (Perfetti et al., 2007).

Finalmente, el modelo propuesto por Perfetti (Perfetti, 1999; Perfetti et al., 2007) coincide con el modelo de Kintsch (Kintsch, 1998; Kintsch y Rawson 2005), principalmente en que la representación más completa del contenido del texto es el modelo de situación. También coinciden en los niveles de procesamiento necesarios para llegar a la conformación de un modelo mental o modelo de situación: nivel de palabra (procesamiento léxico), nivel de oración (procesamiento sintáctico) y nivel textual. Sin embargo, el modelo de Perfetti (Perfetti, 1999; Perfetti et al., 2007) no considera aspectos de arquitectura cognitiva, y presenta un modelo en el marco de "a general framework that exposes the processes of comprehension without making strong assumptions about constraints on their interactions" (Perfetti et al., 2007: 228).

\subsection{DESARROLLO DE LA LECTURA}

El desarrollo de la lectoescritura es un proceso que se inicia muy temprano en la vida de un niño, en el que participan diversos factores y estímulos y que se relaciona estrechamente con la adquisición del lenguaje. Es así como muchos de los factores que participan de las etapas iniciales en el aprendizaje de la lectura inciden en el desempeño de la comprensión lectora en etapas posteriores.

El desarrollo de las habilidades de lectura y escritura es una tarea compleja, compuesta por una serie de procesos cognitivos y determinada por una multiplicidad de factores. La evidencia indica que la alfabetización comienza mucho antes de la escolaridad, ya que los niños, desde la lactancia, "adquieren un conocimiento funcional de los componentes, productos y usos del sistema de escritura y de las formas en las que las actividades de lectura y escritura se relacionan, y se diferencian del lenguaje hablado" (Villalón, 2008: 30).

Dado que todos los sistemas de escritura representan aspectos de una lengua hablada, quien aprende a leer debe conectar las representaciones ortográficas con las representaciones fonológicas y morfológicas correspondientes y, a su vez, con los significados y pronunciaciones que ya conoce. Es así como, entre los aspectos relevantes en el aprendizaje de la lectura, están la conciencia fonológica y la comprensión del vocabulario en el lenguaje oral (Wagner et al., 2006).

Una de las corrientes que respaldan el punto de vista de que la conciencia fonológica se relaciona causalmente con aprender a leer es el modelamiento causal de información longitudinal correlacional. A partir de diversas investigaciones referidas por Wagner et al. (1997), Wagner y Torgesen (1987), de Jong y van der Leij (2002), Perfetti et al. (1988, Cit. en Wagner et al., 2006) se concluye que la relación causal entre variables de desarrollo fonológico y de lectura a nivel de palabra es bidireccional. Por una parte, 
las diferencias individuales en la conciencia fonológica temprana juegan un rol causal sobre las subsiguientes diferencias individuales en las habilidades de lectura a nivel de palabras. Por otra parte, las diferencias individuales en el reconocimiento temprano de grafemas juegan un rol causal sobre las consecutivas diferencias individuales de conciencia fonológica.

Desde otro flanco, es posible establecer una relación causal entre la comprensión del vocabulario en el lenguaje oral y la comprensión de lectura. Se han propuesto tres hipótesis (Anderson y Freebody 1981; Cit. en Wagner et al., 2006):

- la hipótesis instrumental, que afirma que el hecho de conocer más palabras mejora la comprensión lectora;

- la hipótesis del conocimiento, que indica que tanto las diferencias individuales en vocabulario como las que se producen en comprensión lectora son causadas por diferencias individuales en conocimiento conceptual; y

- la hipótesis de aptitud, que dice que el vocabulario y la comprensión lectora están correlacionadas, porque ambas tienen su origen, al menos en parte, en las diferencias individuales en habilidades fundamentales, como la habilidad verbal. Nagy (2007) actualiza esta hipótesis, afirmando que las diferencias individuales en la conciencia metalingüística están causalmente relacionadas con las variables previamente mencionadas.

A partir de la prueba empírica de estas hipótesis, en forma de cuatro modelos causales (Wagner et al., 2007), se puede concluir que las relaciones recíprocas entre desarrollo de vocabulario y comprensión de lectura también se reflejan en las relaciones entre conciencia fonológica y lectura a nivel de palabra. En ambos casos, el desarrollo de las habilidades lingüísticas orales y el de las escritas parecen influenciarse mutuamente de forma beneficiosa.

Lo anterior es reafirmado por Villalón (2008), quien sostiene que los procesos sintácticos e inferenciales, el conocimiento de base y el nivel de vocabulario son comunes a la comprensión oral y la comprensión lectora. Se ha observado una correlación muy alta entre ambas, tanto en estudiantes avanzados como en adultos, lo que indica que la habilidad de extraer significado a partir de un texto escrito demanda el desarrollo de estas competencias en el lenguaje oral.

Como refiere Villalón (2008), algunos estudios han establecido una asociación positiva entre el aumento del vocabulario y el desarrollo de la conciencia fonológica en niños menores de cinco años (Sénéchal et al., 2006; Biemiller, 2006; Cit. en Villalón, 2008). Esta relación explicaría los resultados obtenidos por investigaciones de seguimiento, los que indican que el mayor nivel de vocabulario al inicio de kindergarten tiene un valor predictivo sobre el desarrollo de la conciencia fonológica. Esta predicción es mayor en el caso del lenguaje expresivo que en el receptivo.

Entre los factores predictivos del aprendizaje inicial de la lectura reconocidos por Scaraborough (1998, Cit. en Villalón, 2008) se encuentran el vocabulario, la memoria de palabras y oraciones, el conocimiento de lo impreso, la conciencia fonológica y el conocimiento del alfabeto.

La relación entre el desarrollo del lenguaje oral y el aprendizaje de la lectura es, sin duda, compleja. Un estudio realizado por Torgesen et al. (1997, Cit. en Villalón 2008) concluye que para el $20 \%$ de los estudiantes de cuarto año básico con el rendimiento más bajo en lectura, las pruebas de procesamiento fonológico fueron el mejor predictor, mientras que para el $80 \%$ restante, con mejor desempeño lector, el vocabulario fue mejor 
predictor de su desempeño. Además, un estudio realizado por Dickinson y McCabe (2003, Cit. en Villalón, 2008) demuestra que el aprendizaje de la lectura influye en el desarrollo del lenguaje oral.

En síntesis, entre los factores predictores del desarrollo posterior de las habilidades de producción y comprensión escrita se encuentran la adquisición del vocabulario, la conciencia fonológica y el conocimiento de base. Por lo tanto, la etapa en la que los niños toman conciencia de la existencia del lenguaje escrito, en la que juegan a leer y a escribir, es fundamental, ya que funciona como predictor del aprendizaje posterior. Esto ocurre porque las actividades de conciencia y exploración le permiten al niño comprender la capacidad de comunicar mensajes a través de la escritura.

\subsection{MODELO DE EVALUACIÓN DE LA COMPRENSIÓN LECTORA}

El modelo de evaluación de la comprensión lectora propuesto por Riffo y Véliz (Riffo y Véliz 2011, Véliz y Riffo 1993) pretende entregar parámetros de medición que permitan clasificar a los lectores de acuerdo con el nivel alcanzado en las distintas dimensiones que implica el complejo proceso de la comprensión de lectura. La finalidad de este modelo es guiar la construcción de un instrumento de evaluación que integre los diversos aspectos que participan en el proceso lector.

Las bases teóricas del modelo se encuentran en la psicolingüística, estudios del discurso y la pragmática. Su sustento psicolingüístico está orientado principalmente desde los modelos de comprensión vigentes (principalmente, el modelo de construcción e integración de Kintsch, 1998), lo que es apoyado por el concepto de género discursivo. A partir de lo anterior, se considera que el proceso de comprensión lectora implica un lector, un texto y su contexto, los que interactúan de forma dinámica, ya sea los tres en su conjunto o dos de ellos (lector- texto, texto - contexto, lector - contexto). De esta manera, se puede focalizar la mirada por separado a estas interacciones, para así identificar habilidades específicas.

El modelo de evaluación engloba tres dimensiones de la comprensión lectora. Entre ellas se encuentran la comprensión textual, que corresponde a criterios determinados por el nivel de procesamiento requerido por la tarea; la comprensión pragmática, que especifica criterios determinados por el contexto; y la comprensión crítica, cuyos criterios son determinados por el lector y su posición frente al texto y sus contextos.

La comprensión textual mide la habilidad del lector para resolver tareas en distintos niveles de organización, desde la palabra hasta el texto en su conjunto. Esta dimensión incluye tres niveles:

Nivel proposicional, en el que se miden dos aspectos a) la comprensión de palabras poco frecuentes con dos indicadores: recuperar o reconocer significado a través de claves extraídas del texto, donde la información se encuentra explícita, y generar significado a partir de información implícita en el texto; y b) la comprensión de oraciones con dos indicadores: identificar o relacionar predicados y argumentos e identificar circunstancias, en ambos casos la información puede encontrarse explícita o implícita en el texto.

Nivel microestructural, en el que se mide la comprensión de secuencias de dos oraciones a partir de tres indicadores: a) establecer relaciones de coherencia referencial, b) establecer relaciones de coherencia condicional (que incluyen relaciones temporales y causales), c) establecer relaciones de coherencia funcional (es decir, comprender cómo 
operan los organizadores textuales). En los tres casos la información puede estar explícita o implícita en el texto.

Nivel macroestructural y superestructural, en el que se mide la comprensión de secuencias de más de dos oraciones, párrafos y textos a través de cuatro indicadores: a) reconocer o derivar el significado global del texto, b) establecer relaciones de coherencia condicional, c) establecer relaciones de coherencia funcional y d) determinar la organización lógica del texto (es decir, realizar operaciones relacionadas con el procesamiento de la superestructura). En los cuatro casos, la información puede encontrarse de manera explícita o implícita en el texto.

La comprensión pragmática mide la habilidad del lector para establecer relaciones entre el texto y su contexto, tanto inmediato como cultural. Esta dimensión se vale de cuatro indicadores: a) reconocer los participantes en el círculo comunicativo del texto (distinguir entre enunciado y enunciación), b) situar el texto en el contexto inmediato y en el contexto cultural, c) determinar los propósitos del texto y d) establecer el sentido del texto (es decir, qué sentido adquiere de acuerdo con el contexto en el que circula). En los cuatro aspectos, la información puede encontrarse explícita o implícita en el texto.

La comprensión crítica mide la capacidad de análisis del lector, lo que implica procesamientos metacognitivos y reflexivos a nivel textual. Esta dimensión es evaluada a partir de cuatro indicadores: a) transferir información (recontextualizar información, utilizar la información para resolver problemas y realizar otras actividades de naturaleza reflexiva), b) emitir juicio de valor, c) discutir (es decir, dialogar con el texto) y d) comprender significados no convencionales y operaciones retóricas del discurso (lenguaje figurado). En los cuatro aspectos la información puede estar explícita o implícita en el texto.

Finalmente, el modelo de evaluación de la comprensión lectora propuesto por Riffo y Véliz (Riffo y Véliz 2011, Véliz y Riffo 1993) permite reconocer los distintos factores que inciden en el proceso de la lectura y de la comprensión. Entonces, este modelo, además de permitir entender de mejor manera cómo ocurre el proceso de la lectura, posibilita la aplicación de sus criterios a instrumentos de evaluación ya existentes y verificar los niveles de comprensión lectora que miden.

\section{ANÁLISIS DE LA PRUEBA CLP}

\subsection{METODOLOGÍA DE ANÁLISIS}

Para estudiar la prueba de comprensión lectora de complejidad lingüística progresiva (CLP) se realizó un análisis cualitativo, en el cual se aplicó el modelo de evaluación de comprensión lectora propuesto por Riffo y Véliz (Riffo y Véliz 2011, Véliz y Riffo 1993), lo que fue complementado con el marco teórico presentado y contrastado con lo declarado por los autores de la prueba.

Se clasificaron las preguntas de las formas paralelas de los niveles cuatro y ocho a partir de las dimensiones y niveles propuestos en el modelo de evaluación de la comprensión lectora de Riffo y Véliz (Riffo y Véliz 2011, Véliz y Riffo 1993). Una vez realizada 
la clasificación de las preguntas, se compararon las dos formas para cada nivel, con el fin de determinar si son equivalentes.

\subsection{DESCRIPCIÓN DE LA PRUEBA CLP}

CLP es una prueba estandarizada que mide la comprensión lectora en ocho niveles, coincidentes con los ocho cursos de enseñanza básica en Chile. Para ello, se construyeron textos considerados adecuados para cada nivel, en los que se introdujo una creciente dificultad lingüística. A partir de cada texto, se formula una serie de subtest, que por prueba van de los cuatro a los seis.

El instrumento de evaluación está dividido en niveles y no en cursos, ya que existe conciencia de que en ciertos contextos educacionales el avance en materia de comprensión lectora puede ser más lento. La finalidad de la prueba es arrojar un diagnóstico y orientar el proceso de enseñanza de la lectura. Cada nivel cuenta con dos formas paralelas.

En el caso del nivel cuatro, cada forma de la prueba está conformada por tres textos, desde los que se desprenden cuatro subtest. Ambas formas tienen en común dos de los textos: el primero, una fábula titulada "El pinito descontento"; y el tercero, un cuento llamado "La ballena y el vigía". La medición de la comprensión del primer texto se realiza a través de un subtest de preguntas de comprensión de selección múltiple; en el caso del tercer texto, a través de dos subtest, uno de preguntas de comprensión de selección múltiple y otro de manejo léxico con términos pareados. Las preguntas son distintas para cada forma.

Las formas difieren solo en el segundo texto. Para la forma "A", el segundo texto es el cuento/texto pedagógico "Un viajero espacial" y para la B es el texto pedagógico "Días de aprendizaje". Para ambas formas, el texto es seguido por un subtest de preguntas de comprensión de selección múltiple.

Los autores de CLP describen estos textos como de cierta complejidad, en los que se utilizan moderadamente elementos deícticos y anafóricos, se evita la ambigüedad y se abordan temas literarios y científicos simples. Se espera que, en este nivel, el lector domine las siguientes habilidades: interpretación de elementos deícticos y anafóricos, globalización de la información, distinción entre hechos y opiniones, categorización simple de objetos y personas, establecimiento de relaciones de causa - efecto (Alliende et al., 2004)

El nivel ocho está compuesto por dos textos, comunes para ambas formas, una reseña histórica, "Las variadas pinturas de los mexicanos", y un texto pedagógico, "El hombre y el cielo". En el caso del primer texto, la comprensión se mide a partir de tres subtest: el primero y el segundo corresponden a manejo léxico a través de términos pareados y el tercero está compuesto por seis preguntas de comprensión de selección múltiple. En el segundo texto, la medición de la comprensión se realiza a través de tres subtest: en el primero, se evalúa el manejo léxico a través de siete preguntas de selección múltiple; en el segundo, se mide la comprensión de expresiones poco frecuentes a través de términos pareados y, en el tercero, se presentan seis preguntas de comprensión de opción múltiple.

De acuerdo con la descripción de los autores de CLP, en el texto "Las variadas pinturas de los mexicanos" se observa un cierto orden temporal, con referencias de tipo abstracto, las que se encuentran alejadas en el tiempo y el espacio de los posibles lectores. En el texto "El hombre y el cielo" se remplaza la secuencia temporal por una secuencia 
lógica, lo que promueve la reflexión, hecho que viene dándose en forma progresiva a lo largo de las pruebas.

En este nivel, ya que corresponde al último, se espera que el lector sea capaz de entender textos de mayor extensión, con estructuras variadas y complejas, y con temáticas alejadas de la vida diaria (Alliende et al., 2004).

\subsection{FUNDAMENTOS DE LA PRUEBA CLP}

Los autores de CLP parten del supuesto de que la comprensión lectora puede ser enseñada y medida. De esta manera, en la medición de la comprensión lectora a partir de esta prueba, se consideran dos aspectos clave: determinar diversos niveles de complejidad surgidos del texto y destinar textos específicos a grupos con características comunes (edad cronológica, nivel de escolaridad, etapa de aprendizaje de la lectura, entre otras). Es así como para "enseñar, desarrollar y evaluar la comprensión de la lectura se requiere un adecuado conocimiento del grupo de lectores y un estricto control de la complejidad de los textos que se utilicen" (Alliende et al., 2004:19).

Para determinar la complejidad de los textos escritos, los autores indican que se pueden considerar tres aspectos: sintácticos, semánticos y pragmáticos. Los primeros se refieren al léxico y las estructuras morfosintácticas; los segundos, a los contenidos del texto, en particular, a las relaciones entre las informaciones incluidas en el texto que lo proveen de cohesión y coherencia; y los terceros, a los conocimientos de mundo que posee el lector. Para medir la comprensión lectora a partir de estos tres aspectos, se consideran los elementos que se estiman indispensables para dotar de sentido al texto.

Finalmente, los textos utilizados en la prueba fueron diseñados especialmente para ella, y la selección de los textos con sus respectivas pruebas fue realizada tras varias aplicaciones experimentales. A estas últimas les siguió una serie de análisis estadísticos.

3.4. APLICACIÓN DEL MODELO DE EVALUACIÓN DE LA COMPRENSIÓN LECTORA DE RIFFO Y VÉLIZ (RIFFO Y VÉLIZ 2011, VÉLIZ Y RIFFO 1993) A LA PRUEBA CLP

El modelo de evaluación de la comprensión lectora Riffo y Véliz (Riffo y Véliz 2011, Véliz y Riffo 1993) fue aplicado a los niveles cuatro y ocho de CLP, a cada una de sus formas. Se identificó a qué dimensión de la comprensión lectora corresponde cada pregunta, de acuerdo con los criterios e indicadores propuestos por los ya mencionados autores.

\subsubsection{Aplicación del modelo de evaluación al nivel cuatro de CLP}

El nivel cuatro de CLP está dividido en cuatro subtest. El primero mide la comprensión del texto narrativo "El pinito descontento" y cuenta con cuatro preguntas. En la Tabla 1 se observa el detalle de su clasificación. En ella, se puede apreciar que la forma A se 
Tabla 1. Clasificación de las preguntas del primer sub test, correspondiente a "El pinito descontento"

\begin{tabular}{|c|c|c|c|}
\hline \multicolumn{2}{|c|}{ FORMA A } & \multicolumn{2}{c|}{ FORMA B } \\
\hline Pregunta & Clasificación & Pregunta & Clasificación \\
\hline 1 & $\begin{array}{c}\text { Comprensión textual, nivel } \\
\text { proposicional, comprensión } \\
\text { de oraciones: identificar } \\
\text { predicados y argumentos }\end{array}$ & 1 & $\begin{array}{c}\text { Comprensión crítica: } \\
\text { comprender significados no } \\
\text { convencionales y operaciones } \\
\text { retóricas. }\end{array}$ \\
\hline 2 & $\begin{array}{c}\text { Comprensión textual, nivel } \\
\text { macroestructural: establecer } \\
\text { relaciones de coherencia } \\
\text { condicional. }\end{array}$ & 2 & $\begin{array}{c}\text { Comprensión crítica: transferir } \\
\text { información. }\end{array}$ \\
\hline 3 & $\begin{array}{c}\text { Comprensión textual, nivel } \\
\text { macroestructural: establecer } \\
\text { relaciones de coherencia } \\
\text { condicional. }\end{array}$ & 3 & $\begin{array}{c}\text { Comprensión crítica: emitir } \\
\text { juicio de valor. }\end{array}$ \\
\hline 4 & $\begin{array}{c}\text { Comprensión crítica: } \\
\text { comprender significados } \\
\text { no convencionales y } \\
\text { operaciones retóricas. }\end{array}$ & 4 & $\begin{array}{c}\text { Comprensión textual, nivel } \\
\text { microestructural: establecer } \\
\text { relaciones de coherencia } \\
\text { condicional. }\end{array}$ \\
\hline
\end{tabular}

centra principalmente en la comprensión textual, mientras la forma B se centra en la comprensión crítica, lo que muestra un desequilibrio en lo que mide esta parte de la prueba.

El segundo subtest mide la comprensión de "Un viajero espacial" para la forma A y "Días de aprendizaje" para la forma B. Si bien cada texto aborda un tema distinto, tienen en común la incorporación de secuencias descriptivas basadas en datos científicos (características de las estrellas en el primero y características de los hipopótamos en el segundo). En la Tabla 2 se observa el detalle de la clasificación de las siete preguntas que componen este sub test. Como se puede apreciar, en ambas formas las preguntas se centran en la comprensión textual, principalmente en el nivel proposicional. El nivel

Tabla 2. Clasificación de las preguntas del segundo sub test

\begin{tabular}{|c|c|c|c|}
\hline \multicolumn{2}{|c|}{$\begin{array}{c}\text { FORMA A } \\
\text { "Un viajero espacial" }\end{array}$} & \multicolumn{2}{c|}{$\begin{array}{c}\text { FORMA B } \\
\text { "Días de aprendizaje" }\end{array}$} \\
\hline Pregunta & Clasificación & Pregunta & Clasificación \\
\hline 1 & $\begin{array}{c}\text { Comprensión textual, nivel } \\
\text { proposicional, comprensión } \\
\text { de oraciones: identificar } \\
\text { predicados y argumentos }\end{array}$ & 1 & $\begin{array}{c}\text { Comprensión textual, nivel } \\
\text { proposicional, comprensión de } \\
\text { oraciones: identificar predicados } \\
\text { y argumentos }\end{array}$ \\
\hline
\end{tabular}




\begin{tabular}{|c|c|c|c|}
\hline 2 & $\begin{array}{c}\text { Comprensión textual, nivel } \\
\text { microestructural: establecer } \\
\text { relaciones de coherencia } \\
\text { condicional. }\end{array}$ & 2 & $\begin{array}{c}\text { Comprensión textual, nivel } \\
\text { microestructural: establecer } \\
\text { relaciones de coherencia } \\
\text { condicional. }\end{array}$ \\
\hline 3 & $\begin{array}{c}\text { Comprensión textual, nivel } \\
\text { proposicional, comprensión } \\
\text { de palabras poco frecuentes: } \\
\text { generar significado. }\end{array}$ & 3 & $\begin{array}{c}\text { Comprensión textual, nivel } \\
\text { proposicional, comprensión de } \\
\text { oraciones: identificar predicados } \\
\text { y argumentos }\end{array}$ \\
\hline 4 & $\begin{array}{c}\text { Comprensión textual, nivel } \\
\text { proposicional, comprensión } \\
\text { de oraciones: identificar } \\
\text { predicados y argumentos }\end{array}$ & 4 & $\begin{array}{c}\text { Comprensión textual, nivel } \\
\text { proposicional, comprensión de } \\
\text { oraciones: identificar predicados } \\
\text { y argumentos }\end{array}$ \\
\hline 5 & $\begin{array}{c}\text { Comprensión textual, nivel } \\
\text { proposicional, comprensión } \\
\text { de oraciones: identificar } \\
\text { predicados y argumentos }\end{array}$ & 5 & $\begin{array}{c}\text { Comprensión textual, nivel } \\
\text { proposicional, comprensión de } \\
\text { oraciones: identificar predicados } \\
\text { y argumentos }\end{array}$ \\
\hline 6 & $\begin{array}{c}\text { Comprensión textual, nivel } \\
\text { proposicional, comprensión } \\
\text { de oraciones: identificar } \\
\text { predicados y argumentos }\end{array}$ & 6 & $\begin{array}{c}\text { Comprensión textual, nivel } \\
\text { proposicional, comprensión de } \\
\text { oraciones: identificar predicados } \\
\text { y argumentos }\end{array}$ \\
\hline 7 & $\begin{array}{c}\text { Comprensión textual, nivel } \\
\text { macroestructural: establecer } \\
\text { relaciones de coherencia } \\
\text { condicional. }\end{array}$ & 7 & $\begin{array}{c}\text { Comprensión textual, nivel } \\
\text { microestructural: establecer } \\
\text { relaciones de coherencia } \\
\text { condicional. }\end{array}$ \\
\hline
\end{tabular}

microestructural está presente en ambas formas, y el nivel macroestructural se mide en una de las preguntas de la forma A.

Los dos últimos sub test corresponden a la medición de la comprensión del texto "La ballena y el vigía". En el tercer sub test, en ambas formas las preguntas se centran en el nivel proposicional, específicamente, en la comprensión de oraciones; con excepción

Tabla 3. Clasificación de las preguntas del tercer y del cuarto sub test, correspondientes al texto "La ballena y el vigía"

\begin{tabular}{|c|c|c|c|}
\hline \multicolumn{2}{|c|}{ FORMA A } & \multicolumn{2}{c|}{ FORMA B } \\
\hline Tercer subtest & Clasificación & Pregunta & Clasificación \\
\hline Pregunta & $\begin{array}{c}\text { Comprensión textual, nivel } \\
\text { proposicional, comprensión } \\
\text { de oraciones: identificar } \\
\text { predicados y argumentos }\end{array}$ & & \\
\hline
\end{tabular}




\begin{tabular}{|c|c|c|c|}
\hline 2 & $\begin{array}{l}\text { Comprensión textual, nivel } \\
\text { proposicional, comprensión } \\
\text { de oraciones: identificar } \\
\text { circunstancias. }\end{array}$ & & \\
\hline 3 & $\begin{array}{l}\text { Comprensión textual, nivel } \\
\text { proposicional, comprensión } \\
\text { de oraciones: identificar } \\
\text { circunstancias. }\end{array}$ & & \\
\hline \multicolumn{4}{|c|}{ Cuarto sub test } \\
\hline Pregunta & Clasificación & Pregunta & Clasificación \\
\hline 1 & $\begin{array}{l}\text { Comprensión textual, nivel } \\
\text { proposicional, comprensión } \\
\text { de palabras poco frecuentes: } \\
\text { recuperar significado. }\end{array}$ & 1 & $\begin{array}{c}\text { Comprensión textual, nivel } \\
\text { proposicional, comprensión } \\
\text { de palabras poco frecuentes: } \\
\text { generar significado. }\end{array}$ \\
\hline 2 & $\begin{array}{l}\text { Comprensión textual, nivel } \\
\text { proposicional, comprensión } \\
\text { de palabras poco frecuentes: } \\
\text { recuperar significado. }\end{array}$ & 2 & $\begin{array}{l}\text { Comprensión textual, nivel } \\
\text { proposicional, comprensión } \\
\text { de palabras poco frecuentes: } \\
\text { recuperar significado. }\end{array}$ \\
\hline 3 & $\begin{array}{l}\text { Comprensión textual, nivel } \\
\text { proposicional, comprensión } \\
\text { de palabras poco frecuentes: } \\
\text { recuperar significado. }\end{array}$ & 3 & $\begin{array}{l}\text { Comprensión textual, nivel } \\
\text { proposicional, comprensión } \\
\text { de palabras poco frecuentes: } \\
\text { generar significado. }\end{array}$ \\
\hline 4 & $\begin{array}{l}\text { Comprensión textual, nivel } \\
\text { proposicional, comprensión } \\
\text { de palabras poco frecuentes: } \\
\text { generar significado. }\end{array}$ & 4 & $\begin{array}{l}\text { Comprensión textual, nivel } \\
\text { proposicional, comprensión } \\
\text { de palabras poco frecuentes: } \\
\text { recuperar significado. }\end{array}$ \\
\hline
\end{tabular}

de la tercera pregunta de la forma B, que mide el nivel macroestructural. En cuanto al cuarto sub test, éste se centra en la comprensión de palabras en ambas formas.

En síntesis, en la Tabla 4 se puede observar cuantitativamente la equivalencia de las pruebas, lo que nos permite ver, de manera panorámica y general, en qué niveles de la comprensión lectora se enfoca el nivel cuatro de CLP. A simple vista, es posible reconocer que el número de preguntas por nivel, aunque dispar, es muy similar, por lo que existe equivalencia entre las formas A y B. También se observa que la mayor cantidad 
Tabla 4. Síntesis de la cantidad de preguntas de la prueba CLP nivel 4 por nivel de comprensión

\begin{tabular}{|c|c|c|c|}
\hline \multicolumn{2}{|c|}{ Dimensión de la comprensión lectora } & \multicolumn{2}{|c|}{ Cantidad de preguntas } \\
\hline & & FORMA A & FORMA B \\
\hline \multirow{4}{*}{$\begin{array}{c}\text { COMPRENSIÓN } \\
\text { TEXTUAL }\end{array}$} & $\begin{array}{l}\text { Nivel proposicional } \\
\text { (comp. de palabras) }\end{array}$ & 5 & 4 \\
\hline & $\begin{array}{l}\text { Nivel proposicional } \\
\text { (comp. de } \\
\text { oraciones) }\end{array}$ & 8 & 7 \\
\hline & $\begin{array}{c}\text { Nivel } \\
\text { microestructural }\end{array}$ & 2 & 3 \\
\hline & $\begin{array}{c}\text { Nivel } \\
\text { macroestructural }\end{array}$ & 2 & 1 \\
\hline \multicolumn{2}{|c|}{ LECTURA CRÍTICA } & 1 & 3 \\
\hline
\end{tabular}

de preguntas se concentra en el nivel proposicional, comprensión de palabras y, principalmente, comprensión de oraciones (que abarca alrededor del $40 \%$ de las preguntas).

Finalmente, la equivalencia de las formas A y B del cuarto nivel de CLP se da en especial en los sub test segundo, tercero y cuarto. El primer sub test, sin embargo, muestra diferencias notables entre una forma y otra, ya que la forma A se centra en la evaluación de la comprensión textual, mientras la forma B lo hace en la evaluación de la comprensión crítica.

\subsubsection{Aplicación del modelo de evaluación al nivel ocho de CLP}

La prueba correspondiente al nivel ocho de CLP consta de seis subtest, tres de ellos miden la comprensión de la reseña histórica "Las variadas pinturas de los mexicanos" y los otros tres, la del texto pedagógico "El hombre y el cielo".

En el primer subtest, se evalúa el manejo del vocabulario del estudiante en relación con el texto leído. Se le solicita que empareje las palabras de una columna con las que se encuentran en la columna del frente. En el caso de las primeras cuatro, deben ser emparejadas con un sinónimo escogido entre seis posibilidades. Las siguientes tres deben ser emparejadas con conceptos relacionados. De acuerdo con el modelo de Riffo y Véliz (Riffo y Véliz, 2011; Véliz y Riffo, 1993), este ítem mide la comprensión textual, nivel proposicional, comprensión de palabras poco frecuentes. En el caso de las primeras cuatro, se debe generar significado elaborando un concepto para una palabra usando claves implícitas en el texto. En las tres siguientes, se debe recuperar significado usando claves explícitas del texto.

En el segundo subtest se mide la comprensión textual a nivel proposicional, en particular la comprensión de oraciones (identificar predicados). El estudiante debe identificar la pintura referida en el texto con lo que representa, por lo que debe ir a la oración e identificar lo que se dice de la pintura indicada (el predicado en correspondencia con el sujeto). Se solicita al estudiante que identifique el tipo de pintura (listado con letras) con lo que representa (en una lista enumerada). En las actividades 3, 4, 6, 7 de la forma A de 
la prueba, y en las 1, 2, 3, 4, 5 de la forma B los lectores deben identificar el predicado (o parte del predicado) explícito en el texto. En la actividad 2 de la forma A y la 6 de la forma $\mathrm{B}$ los estudiantes deben reconocer un concepto implícito en el predicado de la oración a la que hace referencia cada actividad.

En ambas formas de la prueba se observa una inconsistencia con las preguntas asociadas a la siguiente oración: "También existían pinturas astronómicas o cronológicas que representaban la situación de los astros, el calendario, las fases de la luna y el pronóstico del tiempo para las diversas épocas del año". Los autores de la prueba interpretan la conjunción coordinante "o" como disyuntiva, cuando en este caso expresa equivalencia entre uno y otro término, lo que los lleva a establecer una separación entre pinturas astronómicas y pinturas cronológicas y les atribuyen a cada una características individuales. Sin embargo, en la oración no se dan pistas para establecer esta división, por lo que las actividades 1 y 5 de la forma A y 7 de la forma B no han sido clasificadas, por ser consideradas confusas para los lectores, razón por la cual no miden ningún aspecto de la comprensión lectora con precisión.

El tercer subtest corresponde a seis preguntas de opción múltiple acerca del contenido del texto leído. En la Tabla 5 se observa el detalle de la clasificación de las preguntas. Se puede apreciar un equilibrio en ambas formas, pues la mayoría de las preguntas

Tabla 5. Clasificación de las preguntas del tercer sub test, correspondiente a "Las variadas pinturas de los mexicanos"

\begin{tabular}{|c|c|c|c|}
\hline \multicolumn{2}{|r|}{ FORMA A } & \multicolumn{2}{|r|}{ FORMA B } \\
\hline Pregunta & Clasificación & Pregunta & Clasificación \\
\hline 1 & $\begin{array}{l}\text { Comprensión textual, nivel } \\
\text { macroestructural: establecer } \\
\text { relaciones de coherencia } \\
\text { condicional. }\end{array}$ & 1 & $\begin{array}{l}\text { Comprensión textual, nivel } \\
\text { microestructural: establecer } \\
\text { relaciones de coherencia } \\
\text { referencial. }\end{array}$ \\
\hline 2 & $\begin{array}{l}\text { Comprensión textual, nivel } \\
\text { macroestructural: establecer } \\
\text { relaciones de coherencia } \\
\text { condicional. }\end{array}$ & 2 & $\begin{array}{l}\text { Comprensión textual, nivel } \\
\text { macroestructural: establecer } \\
\text { relaciones de coherencia } \\
\text { condicional. }\end{array}$ \\
\hline 3 & $\begin{array}{l}\text { Comprensión crítica: } \\
\text { transferir información. }\end{array}$ & 3 & $\begin{array}{l}\text { Comprensión textual, nivel } \\
\text { macroestructural: derivar el } \\
\text { significado global del texto. }\end{array}$ \\
\hline 4 & $\begin{array}{l}\text { Comprensión textual, nivel } \\
\text { proposicional: comprensión } \\
\text { de oraciones, identificar } \\
\text { predicados y argumentos. }\end{array}$ & 4 & $\begin{array}{l}\text { Comprensión crítica: emitir } \\
\text { juicio de valor. }\end{array}$ \\
\hline 5 & $\begin{array}{l}\text { Comprensión crítica: emitir } \\
\text { juicio de valor. }\end{array}$ & & \\
\hline 6 & $\begin{array}{l}\text { Comprensión textual, nivel } \\
\text { proposicional: comprensión } \\
\text { de oraciones, identificar } \\
\text { predicados y argumentos. }\end{array}$ & 6 & $\begin{array}{c}\text { Comprensión textual, nivel } \\
\text { proposicional: comprensión de } \\
\text { oraciones, identificar predicados } \\
\text { y argumentos. }\end{array}$ \\
\hline
\end{tabular}


corresponden a comprensión textual, a nivel macroestructural y proposicional, a las que se suman dos preguntas en la forma $\mathrm{A}$ y una en la forma B de comprensión crítica.

Los subtest cuatro, cinco y seis miden la comprensión del texto pedagógico "El hombre y el cielo". El cuarto subtest mide el manejo léxico del lector. Las siete preguntas, en el caso de las dos formas, miden la comprensión textual, a nivel proposicional, particularmente la comprensión de palabras poco frecuentes (generar significado a partir del texto). Se pide al lector que escoja, de entre las alternativas, la palabra que mejor remplace la que se encuentra destacada en el enunciado extraído del texto leído. Para ello, debe considerar el contexto en el que se usa la palabra destacada, de esta manera podrá discriminar la alternativa correcta. Se considera generar significado porque el estudiante debe reconocer cuál es el sentido con el que se usa la palabra y emparejarla con un sinónimo de ese sentido.

El quinto subtest para este texto mide, al igual que el anterior, la comprensión a nivel proposicional, específicamente, comprensión de expresiones poco frecuentes. En este caso, en ambas formas, se presentan al lector dos filas: la primera, de expresiones que aparecen en el texto leído; la segunda, de expresiones equivalentes a las de la primera. El estudiante debe parear cada término de la fila 1 con los de la fila 2. Para ello, debe basarse en el contexto en el que aparecen las expresiones de la fila 1 en el texto, lo que le dará pistas de cuál es su equivalente en la fila 2. Por esta razón, se considera que este ítem corresponde a generar significado.

El sexto subtest consta de seis preguntas de selección múltiple, cuya clasificación se puede observar en la Tabla 6. En el caso de la forma A, las preguntas miden comprensión textual, tres de ellas a nivel proposicional, una de ellas a nivel microestructural y dos de ellas a nivel macroestructural. La forma B mide en su mayoría comprensión textual, tres

Tabla 6. Clasificación de las preguntas del sexto sub test, correspondiente a "El hombre y el cielo"

\begin{tabular}{|c|c|c|c|}
\hline \multicolumn{2}{|c|}{ FORMA A } & \multicolumn{2}{c|}{ FORMA B } \\
\hline Pregunta & Clasificación & Pregunta & Clasificación \\
\hline 1 & $\begin{array}{c}\text { Comprensión textual, nivel } \\
\text { proposicional, comprensión } \\
\text { de oraciones: relacionar } \\
\text { predicados y argumentos. }\end{array}$ & 1 & $\begin{array}{c}\text { Comprensión textual, nivel } \\
\text { macroestructural: establecer } \\
\text { relaciones de coherencia } \\
\text { condicional. }\end{array}$ \\
\hline 2 & $\begin{array}{c}\text { Comprensión textual, nivel } \\
\text { macroestructural: establecer } \\
\text { relaciones de coherencia } \\
\text { condicional. }\end{array}$ & 2 & $\begin{array}{c}\text { Comprensión textual, nivel } \\
\text { proposicional, comprensión de } \\
\text { oraciones: relacionar predicados } \\
\text { y argumentos. }\end{array}$ \\
\hline 3 & $\begin{array}{c}\text { Comprensión textual, nivel } \\
\text { microestructural: establecer } \\
\text { relaciones de coherencia } \\
\text { funcional. }\end{array}$ & 3 & $\begin{array}{c}\text { Comprensión textual, nivel } \\
\text { macroestructural: establecer } \\
\text { relaciones de coherencia } \\
\text { condicional. }\end{array}$ \\
\hline 4 & $\begin{array}{c}\text { Comprensión textual, nivel } \\
\text { proposicional: comprensión } \\
\text { de oraciones, identificar } \\
\text { predicados y argumentos. }\end{array}$ & 4 & $\begin{array}{c}\text { Comprensión textual, nivel } \\
\text { proposicional: comprensión de } \\
\text { oraciones, identificar predicados } \\
\text { y argumentos. }\end{array}$ \\
\hline
\end{tabular}




\begin{tabular}{|c|c|c|c|}
\hline 5 & $\begin{array}{c}\text { Comprensión textual, nivel } \\
\text { proposicional: comprensión } \\
\text { de oraciones, identificar } \\
\text { predicados y argumentos. }\end{array}$ & 5 & $\begin{array}{c}\text { Comprensión crítica: emitir } \\
\text { juicio de valor. }\end{array}$ \\
\hline 6 & $\begin{array}{c}\text { Comprensión textual, nivel } \\
\text { macroestructural: derivar el } \\
\text { significado global del texto. }\end{array}$ & 6 & $\begin{array}{c}\text { Comprensión textual, nivel } \\
\text { proposicional: comprensión de } \\
\text { oraciones, identificar predicados } \\
\text { y argumentos. }\end{array}$ \\
\hline
\end{tabular}

preguntas a nivel proposicional y dos de ellas a nivel macroestructural; a estas se suma una pregunta que mide comprensión crítica.

En síntesis, de acuerdo con lo presentado en la Tabla 7, existe plena equivalencia entre la forma A y la B del nivel ocho de CLP. Se puede afirmar que miden las mismas dimensiones de la comprensión lectora, en los mismos niveles. Finalmente, se observa

Tabla 7. Síntesis de la cantidad de preguntas de la prueba CLP nivel 8 por nivel de comprensión.

\begin{tabular}{|c|c|c|c|}
\hline \multicolumn{2}{|c|}{ Dimensión de la comprensión lectora } & \multicolumn{2}{|c|}{ Cantidad de preguntas } \\
\hline & & FORMA A & FORMA B \\
\hline \multirow{4}{*}{$\begin{array}{c}\text { COMPRENSIÓN } \\
\text { TEXTUAL }\end{array}$} & $\begin{array}{l}\text { Nivel proposicional } \\
\text { (comp. de palabras) }\end{array}$ & 14 & 14 \\
\hline & $\begin{array}{c}\text { Nivel proposicional } \\
\text { (comp. de } \\
\text { oraciones) }\end{array}$ & 12 & 12 \\
\hline & $\begin{array}{c}\text { Nivel } \\
\text { microestructural }\end{array}$ & 1 & 1 \\
\hline & $\begin{array}{c}\text { Nivel } \\
\text { macroestructural }\end{array}$ & 4 & 4 \\
\hline \multicolumn{2}{|c|}{ LECTURA CRÍTICA } & 2 & 2 \\
\hline
\end{tabular}

un predominio en la medición de la comprensión textual en este nivel, en especial a nivel proposicional.

\section{CONCLUSIÓN}

La prueba CLP ha sido un instrumento válido y útil en la evaluación de la comprensión lectora por más de dos décadas. Dada su detallada formulación y su aplicación respaldada por datos estadísticos se ha convertido en un instrumento altamente usado en las instituciones educacionales chilenas. No obstante, el análisis realizado en el presente trabajo da cuenta de vacíos e imprecisiones en la medición de la comprensión lectora, considerando la teoría más reciente (Kintsch, 1998; Kintsch y Rawson 2005; Perfetti et al., 2007).

La medición de la comprensión lectora en CLP se concentra en la dimensión de comprensión textual. Predomina la evaluación del nivel proposicional, esto es, la comprensión 
de palabras y oraciones. Los niveles microestructural y macroestructural son evaluados en menor medida. Se observan pocas preguntas correspondientes a la dimensión de comprensión crítica. Cabe mencionar que no se mide la comprensión pragmática de los textos.

Entre las fortalezas de CLP podemos destacar su enfoque en la medición de la comprensión a nivel de palabras. Esto concuerda con lo que dice la teoría acerca de la importancia del vocabulario en la comprensión de textos y que es un predictor de la habilidad lectora posterior.

Una de sus principales debilidades es que los textos han sido construidos por los evaluadores. Esto significa que se ha desestimado la importancia de que se mida la lectura a partir de textos auténticos, lo que hace que la clasificación de los textos en géneros discursivos sea difícil, ya que carecen de una intención comunicativo - social real. Esta visión descontextualizada de la lectura se opone a una mirada en la que se considera a la lectura como "una actividad situada, enraizada en un contexto cultural (...). Leer y comprender es "participar" en una actividad preestablecida socialmente" (Cassany y Aliagas, 2009: 18).

En el nivel cuatro, la mayoría de las preguntas se concentra en la medición de la comprensión textual, en especial, a nivel proposicional. Se da especial énfasis a la aplicación de dos habilidades: el dominio léxico y el procesamiento sintáctico. Dichas habilidades son fundamentales para cimentar el desarrollo de la comprensión lectora a futuro del individuo, por lo que su medición en cuarto año básico, cuando el niño termina el primer ciclo de enseñanza formal, es pertinente.

No obstante lo anterior, un mínimo de preguntas (dos para la forma A, una para la forma B) mide la comprensión de más de dos oraciones. Esto indica que los resultados que arroja la evaluación a través de CLP se basan, principalmente, en representaciones de secciones o pasajes de cada texto, por lo que no interpretan la comprensión del niño en los niveles más altos. Lo mismo ocurre con la comprensión crítica de la lectura, la tiene una baja presencia en la prueba (1 pregunta para la forma A, 3 para la forma B).

En el nivel 8, llama la atención que se mantenga el foco en la medición de la comprensión textual a nivel proposicional. De acuerdo con lo declarado por los autores, la prueba aumenta la dificultad a través de sus niveles, y en este nivel, en particular, se espera que el estudiante comprenda a cabalidad textos más complejos. Lo anterior solo se refleja en los textos seleccionados, mas no en lo pretende medirse de ellos.

El análisis de esta prueba a través del modelo de evaluación de la comprensión lectora de Véliz y Riffo $(1993,2011)$ permite comprobar su aplicabilidad, tanto para el diseño como para la evaluación de instrumentos de medición de la comprensión lectora. Esto se debe a que propone criterios de evaluación que apuntan a los diversos procesos y representaciones de la lectura, basados en las teorías psicolingüísticas vigentes e incorpora elementos relevantes para una mirada ecológica del proceso de comprensión lectora, ya que se sitúa en un sistema social y comunicativo, valiéndose de la teoría de géneros discursivos.

Finalmente, si bien CLP ha sido de gran utilidad a lo largo de las últimas décadas, tanto la teoría psicolingüística como los estudios de la pragmática y del discurso más actuales ponen en evidencia la necesidad de actualización de esta prueba, además del diseño de nuevos instrumentos que midan de manera integral la comprensión lectora en niños y jóvenes en edad escolar. 


\section{REFERENCIAS BIBLIOGRÁFICAS}

Alliende, F., Condemarín, M. y Milicic, N. (2004). Prueba CLP, formas paralelas: Manual para la aplicación de la prueba de comprensión lingüística progresiva, ocho niveles de lectura. Santiago de Chile: Ediciones Universidad Católica de Chile.

Cassany, D. y Marín, C. (2009). Miradas y propuestas sobre la lectura. En D. Cassany (Comp.), Para ser letrados (pp. 13-22). Barcelona: Paidós.

Kintsch, W. (1998). Comprehension. A paradigm for cognition. New York: Cambridge.

Kintsch, W. \& Rawson, K. (2005). Comprehension. En M. J. Snowling \& C. Hulme (Eds.), Science of reading. A handbook (pp. 209-226). Oxford: Blackwell.

MINEDUC (2010). Informe nacional de resultados SIMCE 2009, cuarto y octavo básico. Santiago de Chile: Ministerio de Educación.

MINEDUC (2011). Principales resultados nacionales SIMCE 2010 [en línea]. Web: http://www.agenciaeducacion.cl/wp-content/files_mf/informenacionalderesultadossimce2010247mb.pdf (03/2012).

Nagy, W. (2007). Metalinguistic awareness and the vocabulary - comprehension connection. En R. Wagner, A. Muse \& K. Tannenbaum (Eds.), Vocabulary acquisition: implications for reading comprehension (pp. 52-77). New York: Gilford.

OCDE (2009). PISA 2009 Results: What Students Know and Can Do: Student Performance in Reading, Mathematics and Science [en línea]. Web: http://www.oecd.org/dataoecd/10/61/48852548.pdf $(03 / 2012)$.

Perfetti, C., Landi, N. \& Oakhill, J. (2007). The acquisition of reading comprehension skill. En M. J. Snowling \& C. Hulme (Eds.), Science of reading. A handbook (pp. 227-247). Oxford: Blackwell.

Perfetti, C. (1999). Comprehending written language: A blueprint of the reader. En C. Brown \& P. Hagoort (Eds.), The neurocognition of language (pp. 167-208). New York: Oxford University Press.

Perfetti, C. (1985). Reading ability. New York: Oxford University Press.

Perfetti, C. (1991). Representations and awareness in the acquisition of reading competence. En L. Rieben \& C. Perfetti (Eds.), Learning to read: Basic reaserch and its implications (pp. 33-44). New Jersey: Lawrence Erlbaum.

Riffo, B. y Véliz, M. (2011). Modelo de evaluación de la comprensión lectora. Informe de avance proyecto Fondef D08I1179.

Véliz, M. y Riffo, B. (1993). Comprensión textual: Criterios para su evaluación. RLA, n. 31, 85-100.

Villalón, M. (2008). Alfabetización inicial. Claves de acceso a la lectura y escritura desde los primeros meses de vida. Santiago de Chile: Ediciones UC.

Wagner, R., Piasta, S. \& Torgesen, J. (2006). Learning to read. En M. Traxler \& M. Gernsbacher (Eds.), Handbook of Psycholinguistics (pp. 1111-1142). New York: Academic Press.

Wagner, R., Muse, A. \& Tannenbaum, K. (2007). Promising avenues for better understanding implications of vocabulary development for reading comprehension. En R. Wagner; A. Muse \& K. Tannenbaum (Eds.), Vocabulary acquisition: implications for reading comprehension (pp. 276-292). New York: Gilford. 
